\title{
Genetic basis for female receptivity in Drosophila melanogaster: a diallel study
}

\author{
P. CASARES $\dagger$, M. C. CARRACEDO, R. PIÑEIRO, E. SAN MIGUEL* \& L. GARCIA-FLOREZ \\ Departmento de Genetica, Universidad de Oviedo, Julián Claveria s/n, 33071-Oviedo and *Departamento de Genetica, \\ E.T.S.I.A., Universidad de Lugo, 27002 Lugo, Spain
}

\begin{abstract}
Receptivity of sexually mature, virgin females, measured as the time to copulation, has been investigated. Two independent $5 \times 5$ diallel crosses were carried out, each one with a different male tester genotype. Interactions between male and female genotypes were detected because some lines appeared as dominant in one diallel and recessive in the other. The overall picture emerging from the analyses shows a genetic system for female receptivity characterized by additive genes, with dominance for high receptivity in one of the diallel crosses. This remarkable additive component does not support the action of natural selection favouring females with extreme values of receptivity, rather, the lack of intrasexual female competition for mates is suggested.
\end{abstract}

Keywords: diallel, Drosophila, male-female sexual interaction, receptivity.

\section{Introduction}

There is a large amount of experimentation dealing with diverse aspects of sexual behaviour in Drosophila. Mating depends on a complex interaction between the sexes during which a male displays a repertoire of stimuli towards the female. If the female is receptive, mating can occur. The time elapsed until copulation is both a measure of female receptivity and male courtship efficacy and intensity.

Experimental work with Drosophila melanogaster reveals a genetic basis in male mating success, involving behavioural elements such as mating speed (Parsons, 1964; Fulker, 1966) wing vibration (Wood \& Ringo, 1982), courtship latency (Eastwood \& Burnet, 1977), orientation (Collins \& Hewit, 1984), etc. Despite the great influence most authors attribute to the female in deciding mating, very few studies are available on the genetic determination of female behaviour, where most experimental evidence has been obtained indirectly through artificial selection. Manning (1961) for example, by selecting the first pairs to copulate or the latest in doing so, produced lines with slow and fast mating in which both sexes were affected in their courtship behaviour. However, Manning (1963) was unable to find a response in the same base population when selection for slow mating was applied only in the females, and so the occurrence of additive genetic

†Correspondence. variation for female receptivity remained in doubt. Cook (1973) found increased female receptivity in lines selected in both sexes for mating success between normal females and wingless males. Dow (1976a) found increased female receptivity to yellow males in two independent lines selected for mating success. When these selected lines were subjected to a Cavalli biometrical analysis (Dow, 1977), the genetic model for receptivitiy in an observation period of $1 \mathrm{~h}$ contained additive, dominance and maternal components in one selected line, and additive and dominant sex-linked components in the other line. In both lines, dominance was for low receptivity. As far as we know, no other studies have been carried out.

To find out more about the genetic determination of female receptivity we have carried out a biometrical study be means of a $5 \times 5$ diallel cross between inbred lines. It is known that male characteristics greatly affect the estimates of female receptivity and can be considered an experimental variable. In consequence, the diallel cross was replicated with two different male genotypes, in this way increasing the generality of the results.

\section{Materials and methods}

A population of Drosophila melanogaster caught in a locality of Asturias, Spain, was set up as the reference population. Twenty independent inbred lines were obtained from this population by means of 17 genera- 
tions of brother-sister mating. Five of these lines $(\mathrm{A}, \mathrm{B}$, $\mathrm{C}, \mathrm{D}, \mathrm{E})$ were taken at random as the biological material. Female receptivity was estimated from two independent $5 \times 5$ diallel crosses. In each diallel a different male type was used to test for female receptivity. The Drosophila melanogaster males came from two isofemale lines (M1 and M2) of the same population, also caught in Asturias, but different from the inbred lines. The two diallel crosses were carried out simultaneously in a single block of testing the 25 female genotypes against each male genotype.

The flies were reared in a culture medium made with bakers' yeast ( 20 per cent), sugar ( 5 per cent), agar (1.4 per cent) and propionic acid ( 0.5 per cent). Development was at low-density conditions, at $21.5^{\circ} \mathrm{C}$ and under 12:12 h of light:darkness.

Immediately after eclosion, adults were slightly anaesthetized and sexed, the tester males kept in bottles with food in groups of 10 , and the females individually kept in vials. Female receptivity was measured on day 3 after eclosion. One virgin 3-day-old female was introduced into a small empty vial together with two virgin tester males (M1 or M2) of the same age. No anaesthesia was used. The time elapsed from introduction until copulation was taken as a measure of female receptivity. Vials were continuously observed for $30 \mathrm{~min}$ after which any unmated females were registered as failures. Fifty females were tested for each of the 25 female genotypes obtained in each diallel. All the data were obtained in four consecutive days. Observations were made in the morning in a room at $22^{\circ} \mathrm{C}$.

\section{Statistical analyses}

Not all females mated in the $30 \mathrm{~min}$ period of observation, the number of failures being high in some cells but unimportant in others. We assumed that mating times have a normal continuous underlying distribution, and that observations lasting only $30 \mathrm{~min}$ generate truncated data, the unmated females being the upper bound. This truncated distribution poses statistical difficulty which was overcome as follows. First, several transformations of the mating times were tried to produce the closest approximation of the data distribution to normality. The log transformation of the mating times (in seconds) yielded, in accordance with Dow (1976b), acceptably normally distributed values and the best reduction in the heterogeneity of the cell variances, and thus was applied to the raw data. Then, following Manning (1961), the transformed values $(x)$ were regressed against probit values, $(y)$, and the resulting regression line was used to calculate the mean courtship time and error variance of the total essayed individuals ( 50 females).
The mean values of each $5 \times 5$ diallel cross were subjected to biometrical analyses. The error component was the error variance within individuals or random variation (Hayman, 1954b). Given the small number of inbred lines used in the analysis, it is difficult to make inferences about the wild population from which inbreds derive. Thus, the inbred lines were considered as a non-random sample (fixed model). Under the fixed model, Hayman's (1954b) method of subdividing variance is the more suitable to construct variance ratio tests (Wearden, 1964). The resulting components of variation were analysed following the maternal effect model of Wearden (1964).

\section{Results}

Females displayed lower receptivity in crosses with M1 males than with M2 males. Two different results support this: firstly, the number of unmated females averaged over the $5 \times 5$ crosses were 30 per cent and 15 per cent with M1 and M2 males respectively; and secondly, the cumulative number of matings over time differed greatly with each male type (Fig. 1).

Table 1 shows several preliminary analyses of variance of the two $5 \times 5$ diallel crosses. In both, additive genetic variance for female receptivity is suggested from the highly significant differences between inbred lines. The greater of the two error variances obtained with each male was used in the subsequent diallel analyses (Hayman, 1954b). Since these utilize means rather than raw data, the error variances were divided by 50 , that is, the number of individuals on which they

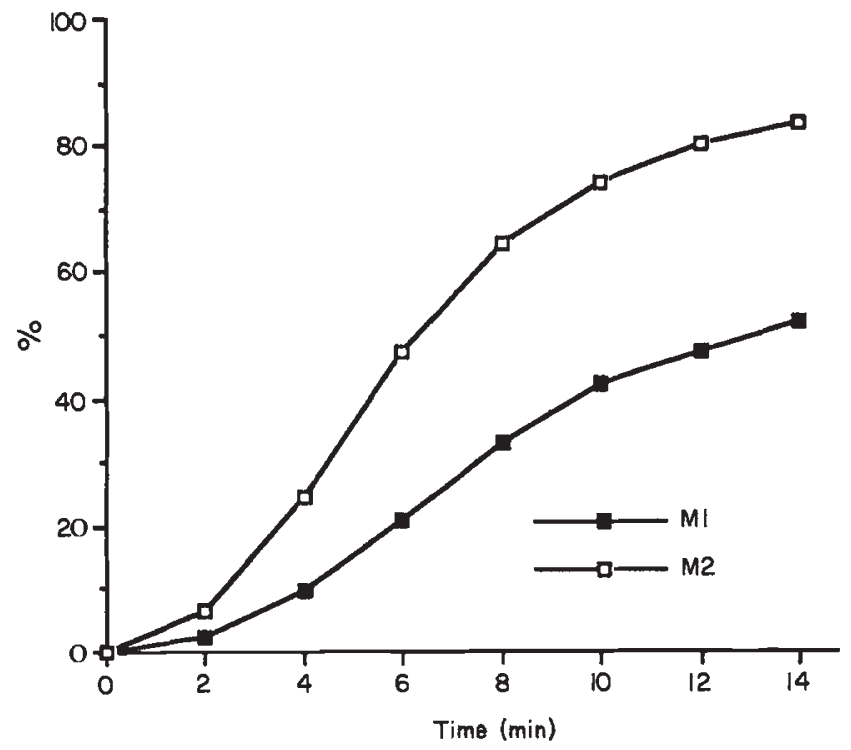

Fig. 1 Cumulative number of matings over time in the dialles with $\mathrm{M} 1$ and $\mathrm{M} 2$ males. 
Table 1 Analyses of variance of courtship times (in decimal log of sec.) observed in inbreds and $\mathrm{F}_{1}$-hybrids in the two $5 \times 5$ diallel crosses

\begin{tabular}{lllrrrr}
\hline Male & & Source of variation & DF & MS $\dagger$ & F & $P$ \\
\hline M1 & Inbreds & Between strains & 4 & 365.37 & 18.38 & $*$ \\
& & Within strains & 245 & 19.88 & & \\
& \multirow{2}{*}{$\mathrm{F}_{1}$ hybrids } & Between crosses & 19 & 168.94 & 8.39 & $*$ \\
& & Within crosses & 980 & 20.13 & & \\
M2 & Inbreds & Between strains & 4 & 119.59 & 5.55 & $*$ \\
& & Within strains & 245 & 21.53 & & \\
& \multirow{2}{*}{$\mathrm{F}_{1}$ hybrids } & Between crosses & 19 & 36.81 & 2.77 & $*$ \\
& & Within crosses & 980 & 13.28 & & \\
\hline
\end{tabular}

$* P<0.001$.

$\dagger$ Multiplied by $10^{-2}$.

are based. In the diallel with the M1 males, the mean of the inbreds compared with the $\mathrm{F}_{1} \mathrm{~s}$ was not different (798 and 856s), whereas in the diallel with the M2 males heterosis was deduced from the faster value in the $\mathrm{F}_{1} \mathrm{~s}$ than the corresponding inbreds (417 and 522s).

\section{Diallel with $M 1$ males}

The results of the diallel analysis with the M1 males appear in Table 2. At the top, the table shows the mean number of mating times backtransformed to seconds; at the bottom, Hayman's subdivision of variance is summarized. The a-item, which tests for additive genetic variation, is highly significant. Dominance is also present, as indicated by the significance of the b-item. Dominance is not directional (b1-item), and all the detected variance is accounted for by the b3-item which reflects dominance effects unique to each $F_{1}$ genotype. No maternal effects are present (c- and d-items).

The variance/covariance analysis is used to test graphically the adequacy of the data with the simple additive/dominance model, and to estimate genetic parameters. The graph corresponding to the diallel cross with M1 males is depicted in Fig. 2. The points fall into a straight line with slope $=0.822 \pm 0.182$, a value which does not differ from unity indicating how well the data fit to the additive/dominance model. Another appropriate test for examining the adjustment of the data to a line of unit slope for non-replicated experiments was devised by Hayman (1954b): applied to our data, the test yields a non-significant $t$-value $(0.61$; d.f. $=3)$ indicating a good fit to the unit slope.

The position of the inbreds on the graph is in accordance with the relative amount of dominant and recessive alleles of each one. Lines $B$ and $C$ carry most
Table 2 Diallel cross with M1 males. Top, mean times of courtship (in $s$ ). Bottom, Hayman's analysis of variance of the data (in decimal $\log$ of $s$ ).

\begin{tabular}{|c|c|c|c|c|c|}
\hline \multirow[b]{2}{*}{ Female lines } & \multicolumn{5}{|c|}{ Male lines } \\
\hline & A & B & $\mathrm{C}$ & $\mathrm{D}$ & E \\
\hline A & 1712 & 1110 & 1455 & 550 & 1319 \\
\hline B & 1284 & 466 & 693 & 497 & 762 \\
\hline $\mathrm{C}$ & 1720 & 476 & 981 & 581 & 696 \\
\hline D & 824 & 392 & 513 & 444 & 744 \\
\hline \multirow[t]{2}{*}{ E } & 1277 & 907 & 700 & 831 & 1324 \\
\hline & \multicolumn{5}{|c|}{ Hayman's analysis } \\
\hline Component & \multicolumn{2}{|c|}{ DF } & \multicolumn{2}{|l|}{$\mathrm{MS} \dagger$} & $\mathrm{F}$ \\
\hline $\mathbf{a}$ & \multicolumn{2}{|c|}{4} & 179.70 & & $44.64^{*}$ \\
\hline b & \multicolumn{2}{|c|}{10} & 13.91 & & $3.45^{*}$ \\
\hline b1 & \multicolumn{2}{|c|}{1} & 3.77 & & 0.94 \\
\hline b2 & \multirow{2}{*}{\multicolumn{2}{|c|}{4}} & 7.03 & & 1.74 \\
\hline b3 & \multirow{2}{*}{\multicolumn{2}{|c|}{$\begin{array}{l}5 \\
4\end{array}$}} & 21.44 & & $5.33^{*}$ \\
\hline c & & & 4.18 & & 1.04 \\
\hline $\mathrm{d}$ & \multirow{2}{*}{\multicolumn{2}{|c|}{6}} & 4.57 & & 1.14 \\
\hline Error & & 325 & 4.02 & & \\
\hline
\end{tabular}

†Multiplied by $10^{-3}$.

$* P<0.001$.

of the genes in which inbreds differ with recessive alleles, whereas the position of lines D and $E$ reflects more dominant than recessive alleles. In accordance with the lack of directional dominance, the phenotypic scores of the inbreds are independent with their respective positions on the graph.

From the parameters obtained in the previous analyses and following the procedure outlined by Hayman (1954b), the average degree of dominance is 


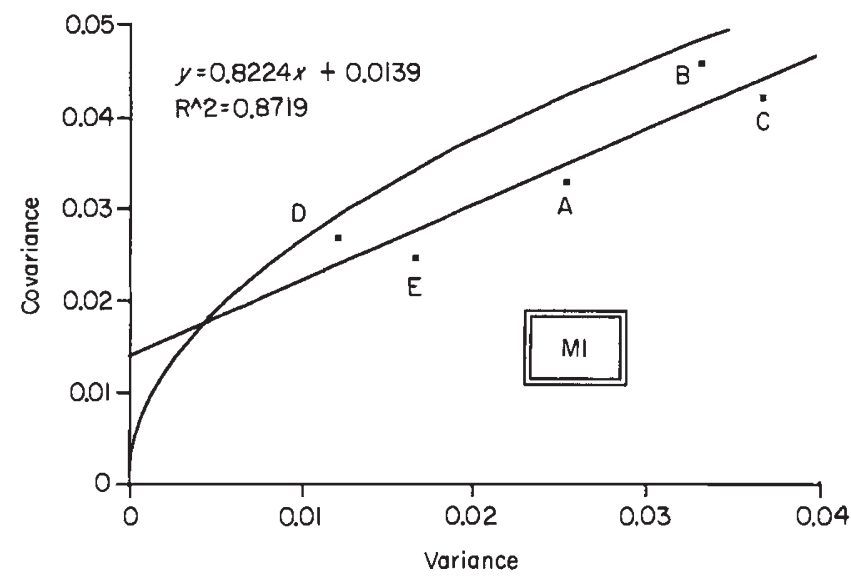

Fig. 2 Variance-covariance graph for the $5 \times 5$ diallel cross with $\mathrm{M} 1$ males.

calculated as 0.57 (partial dominance). Also, the ratio $p / q$ is 0.94 indicating that equal frequencies of dominant and recessive alleles occur in the inbreds.

\section{Dialle/ with M2 males}

The results of the diallel analysis of the $5 \times 5$ genotypes with M2 males are shown in Table 3. The a-item, which is highly significant, reveals important additive genetic variation for female receptivity. Dominance is also present, as shown by the significance of the b-item, being mainly directional (b1-item). No maternal effects are detected.

The graph from the variance/covariance values appears in Fig. 3. The points lie in a straight line of slope $b=0.943 \pm 0.166$, a value that does not differ from unity. Hayman's test indicates the same $(t=0.08$; d.f. $=3$ ). The position of the inbreds on the graph reveals that the $B, C$ and $D$ lines carry most of their genes with dominant alleles, whereas the $\mathrm{A}$ and $\mathrm{E}$ lines carry mainly recessive alleles. The correspondence between the phenotypic scores of the inbreds and their position on the graph indicates that dominance is for highly receptivity.

From the genetic parameters, the average degree of dominance is 0.65 indicating partial dominance, and the ratio $p / q$ is 1.8 reflecting an excess of dominant over recessive alleles in the inbreds.

\section{Discussion}

The analyses of the diallel crosses reveal a mainly additive genetic architecture for female receptivity. The additive component is highly significant with both male genotypes whereas, in comparison, dominance is less important. In the diallel with M1 males, all dominance
Table 3 Diallel cross with M2 males. Top, mean times of courtship (in s). Bottom, Hayman's analysis of variance of the data (in decimal $\log$ of $s$ ).

\begin{tabular}{|c|c|c|c|c|c|}
\hline \multirow[b]{2}{*}{ Female lines } & \multicolumn{5}{|c|}{ Male lines } \\
\hline & A & B & $\mathrm{C}$ & $\mathrm{D}$ & $\mathrm{E}$ \\
\hline A & 807 & 609 & 598 & 366 & 615 \\
\hline B & 444 & 420 & 401 & 391 & 406 \\
\hline C & 373 & 391 & 341 & 362 & 334 \\
\hline $\mathrm{D}$ & 369 & 332 & 311 & 457 & 356 \\
\hline \multirow[t]{2}{*}{$\mathrm{E}$} & 557 & 407 & 438 & 444 & 734 \\
\hline & \multicolumn{5}{|c|}{ Hayman's analysis } \\
\hline Component & \multicolumn{2}{|c|}{ DF } & $\mathrm{MS} \dagger$ & \multicolumn{2}{|r|}{ F } \\
\hline a & \multicolumn{2}{|c|}{4} & 41.18 & \multicolumn{2}{|r|}{$9.56^{* * *}$} \\
\hline b & \multicolumn{2}{|c|}{10} & 8.22 & \multicolumn{2}{|r|}{$1.91^{*}$} \\
\hline $\mathrm{b} 1$ & \multicolumn{2}{|c|}{1} & 38.62 & \multirow{2}{*}{\multicolumn{2}{|c|}{$8.97^{* *}$}} \\
\hline b2 & \multicolumn{2}{|c|}{4} & 6.35 & 1.47 & \\
\hline b3 & \multicolumn{2}{|c|}{5} & 3.63 & \multicolumn{2}{|r|}{0.84} \\
\hline c & \multicolumn{2}{|c|}{4} & 7.55 & \multicolumn{2}{|r|}{1.75} \\
\hline d & \multicolumn{2}{|c|}{6} & 2.92 & \multirow{2}{*}{\multicolumn{2}{|c|}{0.68}} \\
\hline Error & \multicolumn{2}{|c|}{325} & 4.31 & & \\
\hline
\end{tabular}

$\dagger$ Multiplied by $10^{-3}$.

${ }^{*} P<0.05$.

$* * P<0.01$.

${ }^{* * *} P<0.001$.

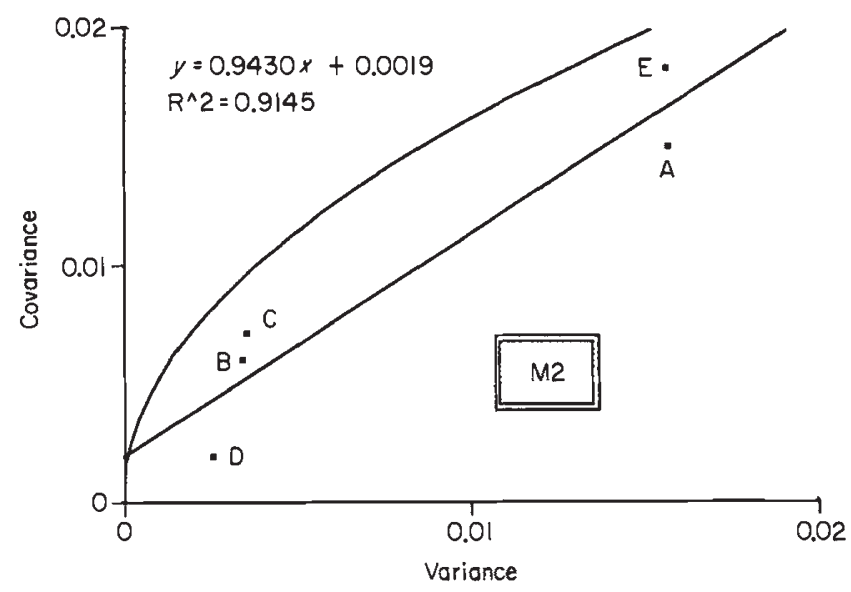

Fig. 3 Variance-covariance graph for the $5 \times 5$ diallel cross with M2 males.

can be explained through specific interactions between the parental and maternal genotypes, dominance being for high receptivity in one cell but for low receptivity in another such as, on average, directional dominance is cancelled. The results with M2 males are different, 
since in this diallel dominance is basically for high receptivity, in clear contrast with the dominance for low receptivity observed by Dow (1977).

It is worth noting that the genetic architecture for female receptivity is dependent on the male genotype against which females were tested. For instance, females from the $E$ line carry mainly additive genes as deduced from the diallel with M1 males, but the same females appear to be clearly recessive in comparison with the other lines in the diallel with $\mathrm{M} 2$ males. On the whole, mating times were shorter with the M2 than the M1 male. It can reasonably be accepted that during courtship M2 males were more effective in lowering the acceptance threshold of the females than the M1 males, which was specially evident in the first minutes, in which females accepted twice the number of M2 than M1 males (Fig. 1).

Although the courtship elements of each male type were not examined, their distinct mating speed suggests that some courtship differences exist between them that females are able to detect. Since male courtship comprises a repertory of different elements (see Spieth \& Ringo 1983) controlled by genetic systems that are, at least partially, different (Collins \& Hewitt, 1984; Gromko, 1987), it seems reasonable to suppose that the female also has different systems of perception and processing with different genetic bases. In accepting the above, the finding of different genetic architectures for receptivity in the same females, when faced with two male types, should not be surprising.

The detection of different genetic architectures depending on the experimental conditions is not rare when traits with complex genetic determination are considered. Examples are given by Wilcock et al. (1981) for escape-avoidance conditioning in rats, and Henderson (1981) for locomotor activity in housemice. In D. melanogaster, Parsons (1964) found a different additive/dominance ratio for male mating speed depending whether the observation period was 10,20 or $40 \mathrm{~min}$. Collins \& Hewitt (1984) found different genetic architectures for some aspects of male courtship in two different designs $(4 \times 4$ diallel or Cavalli's tests), which could presumably be attributed to the use of different female tester genotypes. In a $5 \times 5$ diallel analysis of male mating speed in $D$. melanogaster, P. Casares et al. (unpublished data) found quite different genetic architecture depending on the female tester genotype against which males were examined. In short, mating is a complex behaviour that involves reciprocal interactions between the two sexes. In the present work, we have only studied five female genotypes faced with two kinds of male; with a larger number of genotypes the final result could be much less clear. If we consider that a population consists of several different genotypes then its genetic architecture will be necessarily complex.

Our finding of substantial additive genetic variation in both diallels suggests that selection for increasing or decreasing receptivity should be effective. In terms of evolutionary biology, it is possible to make inferences about the kind of natural selection that has acted in the past (Broadhurst \& Jinks, 1974). If high receptivity is advantageous and directly selected, we expect additive variation to be depleted and analysis to show a dominance component for high receptivity. This is not the case, and several questions can be discussed in this respect. High receptivity could be advantageous if males in the population have a low propensity to mate. In general, males of Drosophila melanogaster are very active sexually, and observations from nature and the laboratory indicate that a female is normally courted by several males simultaneously. This behaviour seems to exclude any possible female competition for mates. That is, receptivity would not be subjected to intrasexual competition. Furthermore, multiple insemination is frequent in D. melanogaster (Pyle \& Gromko, 1981) and can diminish the impact of the first mating on fitness. On the other hand, high receptivity might conflict with the possibility of sexual selection through female choice (Bateman 1948; Spiess 1970), and could represent an increased risk of hybridizing with males of the sibling species $D$. simulans, as we have repeatedly found (Carracedo et al., 1987, 1991).

\section{Acknowledgements}

This work was supported by the Ministry of Education and Science of Spain (DGICYT Grant No. PB870596), and by the University of Oviedo, Spain (COFA Grant No. 89/1606).

\section{References}

BATEMAN, A. J. 1948. Intrasexual selection in Drosophila. Heredity, 2, 349-368.

BROADHURST, P. L. AND JINKS, J. L. 1974. What genetical architecture can tell us about the natural selection of behavioural traits. In: Van Abeelen J. H. F. (ed.) The Genetics of Behaviour North-Holland, Amsterdam, pp. 43-63.

CARRACEDO, M. C., CASARES, P. AND SAN MIGUEL, E. 1987. Sexual isolation between Drosophila melanogaster females and $D$. simulans males. II. Influence of female receptivity on hybridization. Genome, 29, 334-339.

CARRACEDO, M. C., CASARES, P., IZQUIERDO, J. 1. AND PINEIRO, R. 1991. Receptivity and sexual maturation of Drosophila melanogaster females in relation to hybridization with $D$. simulans males. A populational study. Anim. Behav., 42 , 201-208. 
COLLINS, M. F. AND HEWITT, J. K. 1984. The genetic architecture of the male courtship sequence in Drosophila melanogaster. Heredity, 43, 321-337.

CoOK, R. M. 1973. Courtship processing in Drosophila melanogaster. I. Selection for receptivity to wingless males. Anim. Behav., 21, 338-348.

Dow, M. 1976a. Selection for mating success of yellow mutant Drosophila melanogaster: behavioural changes. Behav. Biol., 16, 233-239.

Dow, M. 1976b. Analysis of truncated distributions: mating speed in Drosophila melanogaster. Behav. Genet., 6, 385-389.

Dow, M. 1977. Selection for mating success of yellow mutant Drosophila melanogaster: biometrical genetic analysis. Heredity, 38, 161-168.

EASTWOOD, L. AND BURNET, B. 1977. Courtship latency in male Drosophila melanogaster. Behav. Genet., 7, 359-372.

FULKER, D. W. 1966. Mating speed in male Drosophila melanogaster: a psychogenetic analysis. Science, 153, 203-205.

GROMKO, M. 1987. Genetic constraint on the evolution of courtship behaviour in Drosophila melanogaster. Heredity, 58, 435-441.

HAYMAN, B. 1. 1954a. The analysis of variance of diallel tables. Biometrics, 10, 235-244.

HAYMAN, B. I. 1954b. The theory and analysis of diallel crosses. I. Genetics 39, 789-809.

HENDERSON, N. 1981. Genetic influences on locomotor activity in 11-day-old housemice. Behav. Genet., 11, 209225 .
MANNING, A. 1961. The effects of artificial selection for mating speed in Drosophila melanogaster. Anim. Behav., 9, 82-92.

MANNing, A. 1963. Selection for mating speed in Drosophila melanogaster based on the behavior of one sex. Anim. Behav., 11, 116-120.

PARSONS, P. A. 1964. A diallel cross for mating speeds in Drosophila melanogaster. Genetica, 35, 141-151.

PYLE, D. W. AND GROMKO, M. 1981. Genetic basis for repeated mating in Drosophila melanogaster. Am. Nat., 117, 133-146.

SPIESS, E. B. 1970. Mating propensity and its genetic basis in Drosophila, In: Hecht M. K. and Steere W. C. (eds). Essays in Evolution and Genetics in Honor of Th. Dobzhansky, Appleton-Century-Crofts. New York, pp. 317-379.

SPIETH, H. T. AND RINGO, J. M. 1983. Mating behavior and sexual isolation in Drosophila. In: Ashburner, M., Carson, H. L. and Thompson, J. N. Jr (eds). The Genetics and Biology of Drosophila. Vol. 3C. Academic Press, New York. pp. 223-284.

WEARDEN, s. 1964. Alternative analyses of the diallel cross. Heredity, 19, 669-680.

WILCOCK, J., FULKER, D. W. AND BROADHURST, P. L. 1981. Analysis of two-way escape-avoidance conditioning measures from a diallel cross of eight strains of rats. Behav. Genet., 11, 339-358.

WOOD, D. AND RINGo, J. M. 1982. Artificial selection for altered male wing display in Drosophila simulans. Behav. Genet., 12, 449-458. 